\title{
Thermal Bogoliubov transformation in nuclear structure theory
}

\author{
A. I. Vdovin, Alan A. Dzhioev \\ Bogoliubov Laboratory of Theoretical Physics, Joint Institute for Nuclear Research, \\ 141980 Dubna, Russia
}

\begin{abstract}
Thermal Bogoliubov transformation is an essential ingredient of the thermo field dynamics - the real time formalism in quantum field and many-body theories at finite temperatures developed by $\mathrm{H}$. Umezawa and coworkers. The approach to study properties of hot nuclei which is based on the extension of the well-known Quasiparticle-Phonon Model to finite temperatures employing the TFD formalism is presented. A distinctive feature of the QPM-TFD combination is a possibility to go beyond the standard approximations like the thermal Hartree-Fock or the thermal RPA ones.
\end{abstract}

Among numerous outstanding achievements by N. N. Bogoliubov there is the well-known Bogoliubov transformation for bosons [1] and fermions [2]. This unitary transformation played a crucial role in constructing microscopical theories of superfluidity and superconductivity and till now has been an extremely useful and powerful tool in many branches of theoretical physics. Quite unexpectedly, a new version of the Bogoliubov transformation appeared in the middle of the 1970s when H. Umezawa and coworkers formulated the basic ideas of thermo field dynamics (TFD) [3, 4] - a new formalism extending the quantum field and many-body theories to finite temperatures.

Within TFD [3, 4], the thermal average of a given operator $A$ is calculated as the expectation value in a specially constructed, temperature-dependent state $|0(T)\rangle$ which is termed the thermal vacuum. This expectation value is equal to the usual grand canonical average of $A$. In this sense, the thermal vacuum describes the thermal equilibrium of the system. To construct the state $|0(T)\rangle$, a formal doubling of the system degrees of freedom is introduced 1 . In TFD, a tilde conjugate operator $\widetilde{A}$ - acting in the independent Hilbert space - is associated with $A$, in accordance with properly formulated tilde conjugation rules [3, 4, 5]. For a heated system governed by the Hamiltonian $H$ the whole Hilbert space is spanned by the direct product of the eigenstates of $H$ and those of the tilde Hamiltonian $\widetilde{H}$, both corresponding to the same eigenvalues, i.e. $H|n\rangle=E_{n}|n\rangle$ and $\widetilde{H}|\widetilde{n}\rangle=E_{n}|\widetilde{n}\rangle$. In the doubled Hilbert space, the thermal vacuum is defined as the zero-energy eigenstate

\footnotetext{
${ }^{1}$ It is worth mentioning the general statement [6] that the effect of finite temperature can be included in a free field theory if one doubles the field degrees of freedom.
} 
of the so-called thermal Hamiltonian $\mathcal{H}=H-\widetilde{H}$. Moreover, the thermal vacuum satisfies the thermal state condition [3, 4, 5]

$$
A|0(T)\rangle=\sigma \mathrm{e}^{\mathcal{H} / 2 T} \widetilde{A}^{\dagger}|0(T)\rangle,
$$

where $\sigma=1$ for bosonic $A$ and $\sigma=-i$ for fermionic $A$. It is seen from (11) that, in TFD, there always exists a certain combination of $A$ and $\widetilde{A}^{\dagger}$ which annihilates the thermal vacuum. That mixing is promoted by a specific transformation called the thermal Bogoliubov transformation [4]. This transformation must be canonical in the sense that the algebra of the original system remains the same, keeping its dynamic. The temperature dependence comes from the transformation parameters.

The important point is that in the doubled Hilbert space the time-translation operator is the thermal Hamiltonian $\mathcal{H}$. This means that the excitations of the thermal system are obtained by the diagonalization of $\mathcal{H}$. The existence of the thermal vacuum annihilation operators provides us with a powerful method to analyze physical systems at finite temperatures and allows for straightforward extensions of different zero-temperature approximations.

In the present note, we exemplify advantages of TFD while treating the behavior of atomic nuclei at finite temperatures. In particular, we will show a way of going beyond the thermal RPA and allowing one to treat a coupling of the basic nuclear modes, quasiparticles and phonons [7], at finite temperatures. This problem was already studied in Refs. [8, 9, 10]. However, the new aspects have been revealed recently [11].

To avoid unnecessary complications in the formulae, we consider a nuclear Hamiltonian which is a simplified version of the Hamiltonian of the Quasiparticle-Phonon Model [7]. It consists of a mean field $H_{\mathrm{sp}}$, the BCS pairing interaction $H_{\text {pair }}$, and a separable multipole-multipole particle-hole interaction $H_{\mathrm{ph}}$. Moreover, protons and neutrons are not distinguished. The Hamiltonian reads

$$
\begin{aligned}
& H=H_{\mathrm{sp}}+H_{\mathrm{pair}}+H_{\mathrm{ph}}= \\
& \sum_{j m}\left(E_{j}-\lambda\right) a_{j m}^{\dagger} a_{j m}-\frac{1}{4} G \sum_{j_{1} m_{1} j_{2} m_{2}} a_{j_{1} m_{1}}^{\dagger} a_{\overline{\jmath_{1} m_{1}}}^{\dagger} a_{\overline{j_{2} m_{2}}} a_{j_{2} m_{2}}-\frac{1}{2} \sum_{\lambda \mu} \kappa_{0}^{(\lambda)} M_{\lambda \mu}^{\dagger} M_{\lambda \mu}
\end{aligned}
$$

where $a_{j m}^{\dagger}$ and $a_{j m}$ are the nucleon creation and annihilation operators, $a_{\overline{\jmath m}}=$ $(-1)^{j-m} a_{j-m}$, and $M_{\lambda \mu}^{\dagger}$ is the multipole single-particle operator of the electric type with multipolarity $\lambda$.

At first, we apply TFD to treat pairing correlations at finite temperature (see also [12, 13]). To this aim, we make the standard Bogoliubov $u, v$-transformation from nucleon operators to quasiparticle operators $\alpha^{\dagger}, \alpha$

$$
\begin{aligned}
& \alpha_{j m}^{\dagger}=u_{j} a_{j m}^{\dagger}-v_{j} a_{\overline{\jmath m}}, \\
& \alpha_{j m}=u_{j} a_{j m}-v_{j} a_{\overline{\jmath m}}^{\dagger}\left(u_{j}^{2}+v_{j}^{2}=1\right) .
\end{aligned}
$$


The same transformation with the same $u, v$ coefficients is applied to nucleonic tilde operators $\widetilde{a}_{j m}^{\dagger}, \widetilde{a}_{j m}$, thus producing the tilde quasiparticle operators $\widetilde{\alpha}_{j m}^{\dagger}$ and $\widetilde{\alpha}_{j m}$.

Thermal effects appear after the thermal Bogoliubov transformation which mixes ordinary and tilde quasiparticle operators and produces the operators of so-called thermal quasiparticles $\beta_{j m}^{\dagger}, \beta_{j m}$ and their tilde counterparts. Following the Ojima's formulation of the double tilde conjugation rule for fermions $(\widetilde{\widetilde{a}}=a)[5]$ we use here the complex form of the thermal Bogoliubov transformation:

$$
\begin{aligned}
& \beta_{j m}^{\dagger}=x_{j} \alpha_{j m}^{\dagger}-i y_{j} \widetilde{\alpha}_{j m}, \\
& \widetilde{\beta}_{j m}^{\dagger}=x_{j} \widetilde{\alpha}_{j m}^{\dagger}+i y_{j} \alpha_{j m} \quad\left(x_{j}^{2}+y_{j}^{2}=1\right) .
\end{aligned}
$$

The reasons for this are given in [11].

Then we express the thermal Hamiltonian in terms of thermal quasiparticle operators (4) and require that the one-body part of the thermal BCS Hamiltonian $\mathcal{H}_{\mathrm{BCS}}=H_{\mathrm{sp}}+H_{\text {pair }}-\widetilde{H}_{\mathrm{sp}}-\widetilde{H}_{\text {pair }}$ becomes diagonal in terms of thermal quasiparticles. This yields the following expressions for $u_{j}, v_{j}$ :

$$
u_{j}^{2}=\frac{1}{2}\left(1+\frac{E_{j}-\lambda}{\varepsilon_{j}}\right), \quad v_{j}^{2}=\frac{1}{2}\left(1-\frac{E_{j}-\lambda}{\varepsilon_{j}}\right),
$$

where $\varepsilon_{j}=\sqrt{\left(E_{j}-\lambda\right)^{2}+\Delta^{2}}$. The gap parameter $\Delta$ and the chemical potential $\lambda$ are the solutions of the equations

$$
\Delta=\frac{G}{2} \sum_{j}(2 j+1)\left(x_{j}^{2}-y_{j}^{2}\right) u_{j} v_{j}, \quad N=\sum_{j}(2 j+1)\left(v_{j}^{2} x_{j}^{2}+u_{j}^{2} y_{j}^{2}\right),
$$

where $N$ is the number of nucleons in a nucleus.

With $u_{j}, v_{j}$ from (5) the one-body part of the thermal BCS Hamiltonian reads

$$
\mathcal{H}_{\mathrm{BCS}} \simeq \sum_{j m} \varepsilon_{j}\left(\beta_{j m}^{\dagger} \beta_{j m}-\widetilde{\beta}_{j m}^{\dagger} \widetilde{\beta}_{j m}\right) .
$$

One can see that the Hamiltonian $\mathcal{H}_{\mathrm{BCS}}$ describes a system of noninteracting thermal quasiparticles and tilde-quasiparticles with energies $\varepsilon_{j}$ and $-\varepsilon_{j}$, respectively.

To determine the thermal vacuum corresponding to $\mathcal{H}_{\mathrm{BCS}}$, we need to fix appropriately the coefficients $x_{j}, y_{j}$. In Refs. [12, 13], the coefficients were found by minimizing the thermodynamic potential of the system of noninteracting Bogoliubov quasiparticles. Here we demand that the vacuum $|0(T) ; q p\rangle$ of thermal quasiparticles obey the thermal state condition (1)

$$
a_{j m}|0(T) ; \mathrm{qp}\rangle=-i \mathrm{e}^{\mathcal{H}_{\mathrm{BCS}} / 2 T} \widetilde{a}_{j m}^{\dagger}|0(T) ; \mathrm{qp}\rangle .
$$

Combining (8) and (3) one gets

$$
y_{j}=\left[1+\exp \left(\frac{\varepsilon_{j}}{T}\right)\right]^{-1 / 2}, \quad x_{j}=\left(1-y_{j}^{2}\right)^{1 / 2} .
$$


We see that the coefficients $y_{j}^{2}$ are the thermal Fermi-Dirac occupation factors which determine the average number of thermally excited Bogoliubov quasiparticles in the BCS thermal vacuum. Equations (5), (6), and (9) are the well-known finitetemperature BCS equations [14].

In the next stage we partially take into account the particle-hole residual interaction $H_{\mathrm{ph}}$. Now the thermal Hamiltonian reads

$$
\mathcal{H}=\sum_{j m} \varepsilon_{j}\left(\beta_{j m}^{\dagger} \beta_{j m}-\widetilde{\beta}_{j m}^{\dagger} \widetilde{\beta}_{j m}\right)-\frac{1}{2} \sum_{\lambda \mu} \kappa_{0}^{(\lambda)}\left\{M_{\lambda \mu}^{\dagger} M_{\lambda \mu}-\widetilde{M}_{\lambda \mu}^{\dagger} \widetilde{M}_{\lambda \mu}\right\}
$$

and it can be divided into two parts $-\mathcal{H}_{\mathrm{TQRPA}}$ and $\mathcal{H}_{\mathrm{qph}}$. The part $\mathcal{H}_{\mathrm{TQRPA}}$ that contains $\mathcal{H}_{\mathrm{BCS}}$ and the terms with even numbers of creation and annihilation operators of thermal quasiparticles is approximately diagonalized within the Thermal Quasiparticle Random Phase Approximation, whereas the part $\mathcal{H}_{\mathrm{qph}}$ containing odd numbers of creation and annihilation operators is responsible for the coupling of TQRPA eigenvectors (thermal phonons).

To diagonalize $\mathcal{H}_{\mathrm{TQRPA}}$, the following operator of thermal phonon is introduced:

$$
\begin{aligned}
Q_{\lambda \mu i}^{\dagger}=\frac{1}{2} \sum_{j_{1} j_{2}}\left(\psi_{j_{1} j_{2}}^{\lambda i}\left[\beta_{j_{1}}^{\dagger} \beta_{j_{2}}^{\dagger}\right]_{\mu}^{\lambda}+\widetilde{\psi}_{j_{1} j_{2}}^{\lambda i}\left[\widetilde{\beta}_{\overline{j_{1}}}^{\dagger} \widetilde{\beta}_{\bar{J}_{2}}^{\dagger}\right]_{\mu}^{\lambda}+2 i \eta_{j_{1} j_{2}}^{\lambda i}\left[\beta_{j_{1}}^{\dagger} \widetilde{\beta}_{\bar{J}_{2}}^{\dagger}\right]_{\mu}^{\lambda}\right) \\
+(-1)^{\lambda-\mu}\left(\phi_{j_{1} j_{2}}^{\lambda i}\left[\beta_{j_{1}} \beta_{j_{2}}\right]_{-\mu}^{\lambda}+\widetilde{\phi}_{j_{1} j_{2}}^{\lambda i}\left[\widetilde{\beta}_{\overline{J_{1}}} \widetilde{\beta}_{\overline{J_{2}}}\right]_{-\mu}^{\lambda}-2 i \xi_{j_{1} j_{2}}^{\lambda i}\left[\beta_{j_{1}} \widetilde{\beta}_{\overline{J_{2}}}\right]_{-\mu}^{\lambda}\right),
\end{aligned}
$$

where the notation []$_{\mu}^{\lambda}$ means the coupling of single-particle momenta $j_{1}, j_{2}$ to the angular momentum $\lambda$ with the projection $\mu$. Now the thermal equilibrium state is treated as a vacuum $|0(T) ; \mathrm{ph}\rangle$ for thermal phonons. In addition, the thermal phonon operators are assumed to obey bosonic commutation rules. This imposes some constraints on the phonon amplitudes $\psi, \widetilde{\phi}, \eta$ etc. (see Ref. [11] for more details).

To find eigenvalues of $\mathcal{H}_{\mathrm{TQRPA}}$, the variational principle is applied, i.e. we find the minimum of the expectation value of $\mathcal{H}_{\mathrm{TQRPA}}$ with respect to one-phonon states $Q_{\lambda \mu i}^{\dagger}|0(T) ; \mathrm{ph}\rangle$ or $\widetilde{Q}_{\overline{\lambda \mu i}}^{\dagger}|0(T) ; \mathrm{ph}\rangle$ under afore-mentioned constraints on the phonon amplitudes. As a result we arrive at the following equation for thermal phonon energies $\omega_{\lambda i}$ :

$$
\frac{2 \lambda+1}{\kappa_{0}^{(\lambda)}}=\sum_{j_{1} j_{2}}\left(f_{j_{1} j_{2}}^{(\lambda)}\right)^{2}\left[\frac{\left(u_{j_{1} j_{2}}^{(+)}\right)^{2} \varepsilon_{j_{1} j_{2}}^{(+)}\left(1-y_{j_{1}}^{2}-y_{j_{2}}^{2}\right)}{\left(\varepsilon_{j_{1} j_{2}}^{(+)}\right)^{2}-\omega^{2}}-\frac{\left(v_{j_{1} j_{2}}^{(-)}\right)^{2} \varepsilon_{j_{1} j_{2}}^{(-)}\left(y_{j_{1}}^{2}-y_{j_{2}}^{2}\right)}{\left(\varepsilon_{j_{1} j_{2}}^{(-)}\right)^{2}-\omega^{2}}\right],
$$

where $f_{j_{1} j_{2}}^{(\lambda)}$ is the reduced single-particle matrix element of the multipole operator $M_{\lambda \mu} ; \varepsilon_{j_{1} j_{2}}^{( \pm)}=\varepsilon_{j_{1}} \pm \varepsilon_{j_{2}}, u_{j_{1} j_{2}}^{(+)}=u_{j_{1}} v_{j_{2}}+v_{j_{1}} u_{j_{2}}, v_{j_{1} j_{2}}^{(-)}=u_{j_{1}} u_{j_{2}}-v_{j_{1}} v_{j_{2}}$.

Although at the present stage phonon amplitudes cannot be determined unambiguously, the TQRPA Hamiltonian is diagonal in terms of thermal phonon operators

$$
\mathcal{H}_{\mathrm{TQRPA}}=\sum_{\lambda \mu i} \omega_{\lambda i}\left(Q_{\lambda \mu i}^{\dagger} Q_{\lambda \mu i}-\widetilde{Q}_{\lambda \mu i}^{\dagger} \widetilde{Q}_{\lambda \mu i}\right)
$$


One can see that $\mathcal{H}_{\mathrm{TQRPA}}$ is invariant under the following thermal Bogoliubov transformation:

$$
Q_{\lambda \mu i}^{\dagger} \rightarrow X_{\lambda i} Q_{\lambda \mu i}^{\dagger}-Y_{\lambda i} \widetilde{Q}_{\lambda \mu i}, \quad \widetilde{Q}_{\lambda \mu i}^{\dagger} \rightarrow X_{\lambda i} \widetilde{Q}_{\lambda \mu i}^{\dagger}-Y_{\lambda i} Q_{\lambda \mu i}
$$

with $X_{\lambda i}^{2}-Y_{\lambda i}^{2}=1$. To fix the coefficients $X_{\lambda i}, Y_{\lambda i}$ and finally determine the phonon amplitudes $\psi, \widetilde{\psi}, \phi, \widetilde{\phi}, \eta, \xi$, we again demand that the thermal phonon vacuum obey the thermal state condition ${ }^{2}$. For $A$ in (11), it is convenient to take the multipole operator $M_{\lambda \mu}$. Then the thermal state condition takes the form

$$
M_{\lambda \mu}|0(T) ; \mathrm{ph}\rangle=\mathrm{e}^{\mathcal{H}_{\mathrm{TQRPA}} / 2 T} \widetilde{M}_{\lambda \mu}^{\dagger}|0(T) ; \mathrm{ph}\rangle .
$$

Expressing $M_{\lambda \mu}$ through phonon operators we find the coefficients $X_{\lambda i}, Y_{\lambda i}$

$$
Y_{\lambda i}=\left[\exp \left(\frac{\omega_{\lambda i}}{T}\right)-1\right]^{-1 / 2}, \quad X_{\lambda i}=\left[1+Y_{\lambda i}^{2}\right]^{1 / 2} .
$$

The coefficients $Y_{\lambda i}^{2}$ appear to be the thermal occupation factors of the Bose-Einstein statistics. Thus, the phonon amplitudes are dependent on both the types of thermal occupation numbers: quasiparticle ones (the Fermi-Dirac type) and phonon ones (the Bose-Einstein type). The expressions for all the phonon amplitudes $\psi, \widetilde{\psi}, \phi, \widetilde{\phi}, \eta, \xi$ can be found in [11].

Once the structure of thermal phonons is determined, one can find the $E \lambda$ transition strengths from the TQRPA thermal vacuum to one-phonon states. The transition strengths to non-tilde and tilde one-phonon states are related by

$$
\widetilde{\Phi}_{\lambda i}^{2}=\exp \left(-\omega_{\lambda i} / T\right) \Phi_{\lambda i}^{2} .
$$

This relation is equivalent to the principle of detailed balancing connecting the probabilities for a probe to transfer energy $\omega$ to a heated system and to absorb energy $\omega$ from a heated system.

Now we are ready to go beyond TQRPA and consider the effects of the term $\mathcal{H}_{\mathrm{qph}}$ which is a thermal analogue of the quasiparticle-phonon interaction [7]. It reads

$$
\mathcal{H}_{\mathrm{qph}}=-\frac{1}{2} \sum_{\lambda \mu i} \sum_{j_{1} j_{2}} \frac{f_{j_{1} j_{2}}^{(\lambda)}}{\sqrt{\mathcal{N}^{\lambda i}}}\left\{\left(Q_{\overline{\lambda \mu i}}^{\dagger}+Q_{\lambda \mu i}\right) B_{\lambda \mu i}\left(j_{1} j_{2}\right)+(\text { h.c. })-(\text { t.c. })\right\}
$$

where the notation "(h.c.)" and "(t.c.)" stands for the items which are hermitianand tilde-conjugate to the displayed ones; $\mathcal{N}^{\lambda i}$ is the normalization factor in the phonon amplitudes. The operator $B_{\lambda \mu i}\left(j_{1} j_{2}\right)$ reads

$$
\begin{aligned}
B_{\lambda \mu i}\left(j_{1} j_{2}\right)=i u_{j_{1} j_{2}}^{(+)}\left(\mathcal{Z}_{j_{1} j_{2}}^{\lambda i}\left[\beta_{j_{1}}^{\dagger} \widetilde{\beta}_{j_{2}}\right]_{\mu}^{\lambda}+\mathcal{Z}_{j_{2} j_{1}}^{\lambda i}\left[\widetilde{\beta}_{\overline{J_{1}}}^{\dagger} \beta_{\overline{j_{2}}}\right]_{\mu}^{\lambda}\right)- & \\
& v_{j_{1} j_{2}}^{(-)}\left(\mathcal{X}_{j_{1} j_{2}}^{\lambda i}\left[\beta_{j_{1}}^{\dagger} \beta_{\left.\overline{j_{2}}\right]_{\mu}}^{\lambda}+\mathcal{Y}_{j_{1} j_{2}}^{\lambda i}\left[\widetilde{\beta}_{\overline{j_{1}}}^{\dagger} \widetilde{\beta}_{j_{2}}\right]_{\mu}^{\lambda}\right),\right.
\end{aligned}
$$

\footnotetext{
${ }^{2}$ Earlier, in [1] we have used the other procedure to this aim. That procedure seems to be much less evident and more lengthy.
} 
where the coefficients $\mathcal{X}_{j_{1} j_{2}}^{\lambda i}, \mathcal{Y}_{j_{1} j_{2}}^{\lambda i}$ and $\mathcal{Z}_{j_{1} j_{2}}^{\lambda i}$ are the following:

$$
\left(\begin{array}{l}
\mathcal{X} \\
\mathcal{Y}
\end{array}\right)_{j_{1} j_{2}}^{\lambda i}=x_{j_{1}} x_{j_{2}}\left(\begin{array}{l}
X \\
Y
\end{array}\right)_{\lambda i}+y_{j_{1}} y_{j_{2}}\left(\begin{array}{c}
Y \\
X
\end{array}\right)_{\lambda i}, \quad \mathcal{Z}_{j_{1} j_{2}}^{\lambda i}=x_{j_{1}} y_{j_{2}} X_{\lambda i}+y_{j_{1}} x_{j_{2}} Y_{\lambda i}
$$

The term $\mathcal{H}_{\mathrm{qph}}$ couples states with a different number of thermal phonons. To take into account the phonon coupling, we consider a trial wave function of the following form:

$$
\begin{gathered}
\left|\Psi_{\nu}(J M)\right\rangle=\left[\sum_{i}\left\{R_{i}(J \nu) Q_{J M i}^{\dagger}+\widetilde{R}_{i}(J \nu) \widetilde{Q}_{J M i}^{\dagger}\right\}+\sum_{\substack{\lambda_{1} i_{1} \\
\lambda_{2} i_{2}}}\left\{P_{\lambda_{2} i_{2}}^{\lambda_{1} i_{1}}(J \nu)\left[Q_{\lambda_{1} i_{1}}^{\dagger} Q_{\lambda_{2} i_{2}}^{\dagger}\right]_{M}^{J}\right.\right. \\
\left.\left.+\sum_{\substack{\lambda_{1} i_{1} \\
\lambda_{2} i_{2}}} S_{\lambda_{2} i_{2}}^{\lambda_{1} i_{1}}(J \nu)\left[Q_{\lambda_{1} i_{1}}^{\dagger} \widetilde{Q}_{\overline{\lambda_{2} i_{2}}}^{\dagger}\right]_{M}^{J}+\sum_{\substack{\lambda_{1} i_{1} \\
\lambda_{2} i_{2}}} \widetilde{P}_{\lambda_{2} i_{2}}^{\lambda_{1} i_{1}}(J \nu)\left[\widetilde{Q}_{\overline{\lambda_{1} i_{1}}}^{\dagger} \widetilde{Q}_{\overline{\lambda_{2} i_{2}}}^{\dagger}\right]_{M}^{J}\right\}\right]|0(T) ; \mathrm{ph}\rangle . \quad \text { (17) }
\end{gathered}
$$

It should be stressed that in (17) we keep the thermal vacuum of TQRPA. It means that we do not consider the influence of phonon coupling on thermal occupation numbers. Note also that the function (17) contains not only non-tilde one-phonon components but the tilde ones as well. This is a new point in comparison with Ref. [11].

The function (17) has to be normalized. This demand imposes the following constraint on the amplitudes $R, \widetilde{R}, P, S, \widetilde{P}$ :

$$
\begin{aligned}
\sum_{i}\left\{\left[R_{i}(J \nu)\right]^{2}\right. & \left.+\left[\widetilde{R}_{i}(J \nu)\right]^{2}\right\} \\
& +\sum_{\substack{\lambda_{1} i_{1} \\
\lambda_{2} i_{2}}}\left\{2\left[P_{\lambda_{2} i_{2}}^{\lambda_{1} i_{1}}(J \nu)\right]^{2}+\left[S_{\lambda_{2} i_{2}}^{\lambda_{1} i_{1}}(J \nu)\right]^{2}+2\left[\widetilde{P}_{\lambda_{2} i_{2}}^{\lambda_{1} i_{1}}(J \nu)\right]^{2}\right\}=1 .
\end{aligned}
$$

Since the trial function contains three different types of two-phonon components, there are three types of interaction matrix elements which couple a thermal onephonon state with two-phonon ones

$$
\begin{aligned}
U_{\lambda_{2} i_{2}}^{\lambda_{1} i_{1}}(J i) & =\left\langle 0(T) ; \mathrm{ph}\left|Q_{J M i} \mathcal{H}_{\mathrm{qph}}\left[Q_{\lambda_{1} i_{1}}^{\dagger} Q_{\lambda_{2} i_{2}}^{\dagger}\right]_{M}^{J}\right| 0(T) ; \mathrm{ph}\right\rangle, \\
V_{\lambda_{2} i_{2}}^{\lambda_{1} i_{1}}(J i) & =\left\langle 0(T) ; \operatorname{ph}\left|Q_{J M i} \mathcal{H}_{\mathrm{qph}}\left[Q_{\lambda_{1} i_{1}}^{\dagger} \widetilde{Q}_{\overline{\lambda_{2} i_{2}}}^{\dagger}\right]_{M}^{J}\right| 0(T) ; \mathrm{ph}\right\rangle, \\
W_{\lambda_{2} i_{2}}^{\lambda_{1} i_{1}}(J i) & =\left\langle 0(T) ; \operatorname{ph}\left|Q_{J M i} \mathcal{H}_{\mathrm{qph}}\left[\widetilde{Q}_{\overline{\lambda_{1} i_{1}}}^{\dagger} \widetilde{Q}_{\overline{\lambda_{2} i_{2}}}^{\dagger}\right]_{M}^{J}\right| 0(T) ; \mathrm{ph}\right\rangle .
\end{aligned}
$$

The expressions for the matrix elements $U_{\lambda_{2} i_{2}}^{\lambda_{1} i_{1}}(J i), V_{\lambda_{2} i_{2}}^{\lambda_{1} i_{1}}(J i)$, and $W_{\lambda_{2} i_{2}}^{\lambda_{1} i_{1}}(J i)$ via the phonon amplitudes $\psi, \widetilde{\psi}, \phi$ etc. can be found in [11]. 
Applying the variational principle to the average value of the thermal Hamiltonian $\mathcal{H}_{\mathrm{TQRPA}}+\mathcal{H}_{\mathrm{qph}}$ with respect to $\left|\Psi_{\nu}(J M)\right\rangle$ under the normalization constraint (18) one gets a system of linear equations for the amplitudes $R, \widetilde{R}, P, S, \widetilde{P}$. The system has a nontrivial solution if the energy $\eta_{\nu}$ of the state $\left|\Psi_{\nu}(J M)\right\rangle$ obeys the following secular equation:

$$
\operatorname{det}\left|\begin{array}{cc}
A\left(\eta_{\nu}\right) & B\left(\eta_{\nu}\right) \\
B\left(-\eta_{\nu}\right) & A\left(-\eta_{\nu}\right)
\end{array}\right|=0
$$

where

$$
\begin{aligned}
A_{i i^{\prime}}\left(\eta_{\nu}\right)=\left(\omega_{J i}-\eta_{\nu}\right) \delta_{i i^{\prime}}- & \frac{1}{2} \sum_{\substack{\lambda_{1} i_{1} \\
\lambda_{2} i_{2}}}\left\{\frac{U_{\lambda_{2} i_{2}}^{\lambda_{1} i_{1}}(J i) U_{\lambda_{2} i_{2}}^{\lambda_{1} i_{1}}\left(J i^{\prime}\right)}{\omega_{\lambda_{1} i_{1}}+\omega_{\lambda_{2} i_{2}}-\eta_{\nu}}\right. \\
& \left.+2 \frac{V_{\lambda_{2} i_{2}}^{\lambda_{1} i_{1}}(J i) V_{\lambda_{2} i_{2}}^{\lambda_{1} i_{1}}\left(J i^{\prime}\right)}{\omega_{\lambda_{1} i_{1}}-\omega_{\lambda_{2} i_{2}}-\eta_{\nu}}-\frac{W_{\lambda_{2} i_{2}}^{\lambda_{1} i_{1}}(J i) W_{\lambda_{2} i_{2}}^{\lambda_{1} i_{1}}\left(J i^{\prime}\right)}{\omega_{\lambda_{1} i_{1}}+\omega_{\lambda_{2} i_{2}}+\eta_{\nu}}\right\}
\end{aligned}
$$

and

$$
\begin{aligned}
B_{i i^{\prime}}\left(\eta_{\nu}\right)=\frac{1}{2} \sum_{\substack{\lambda_{1} i_{1} \\
\lambda_{2} i_{2}}}\left\{\frac{U_{\lambda_{2} i_{2}}^{\lambda_{1} i_{1}}(J i) W_{\lambda_{2} i_{2}}^{\lambda_{1} i_{1}}\left(J i^{\prime}\right)}{\omega_{\lambda_{1} i_{1}}+\omega_{\lambda_{2} i_{2}}-\eta_{\nu}}\right. \\
\left.+2(-1)^{\lambda_{1}+\lambda_{2}+J} \frac{V_{\lambda_{2} i_{2}}^{\lambda_{1} i_{1}}(J i) V_{\lambda_{1} i_{1}}^{\lambda_{2} i_{2}}\left(J i^{\prime}\right)}{\omega_{\lambda_{1} i_{1}}-\omega_{\lambda_{2} i_{2}}-\eta_{\nu}}-\frac{W_{\lambda_{2} i_{2}}^{\lambda_{1} i_{1}}(J i) U_{\lambda_{2} i_{2}}^{\lambda_{1} i_{1}}\left(J i^{\prime}\right)}{\omega_{\lambda_{1} i_{1}}+\omega_{\lambda_{2} i_{2}}+\eta_{\nu}}\right\} .
\end{aligned}
$$

Physical effects which can be treated with the function $\left|\Psi_{\nu}(J M)\right\rangle$ and Eq. (20) relate to fragmentation of basic nuclear excitations like quasiparticles and phonons, their spreading widths and/or more consistent description of transition strength distributions over a nuclear spectrum in hot nuclei.

The authors are thankful to Dr. V. Ponomarev for valuable discussions and comments.

\section{References}

[1] Bogoliubov N. N. // Izv. AN SSSR, fizika. 1947. V.11. P.77

[2] Bogoliubov N. N. // JETP. 1958. V.34. P.58

[3] Takahashi Y., Umezawa H.// Coll. Phenom. 1975. V.2. P.55.

[4] Umezawa H., Matsumoto H., Tachiki M. // Thermo field dynamics and condensed states. Amsterdam: North-Holland, 1982. 
[5] Ojima I. // Ann. Phys. 1981. V.137. P.1.

[6] Haag R., Hugenholtz N. W., Winnihk M. // Comm. Math. Phys. 1967. V.5. P.215.

[7] Soloviev V. G. // Theory of Atomic Nuclei: Quasiparticles and Phonons. Bristol: IoP, 1992.

[8] Tanabe K. // Phys. Rev. C. 1988. V.37. P.2802.

[9] Kosov D. S., Vdovin A. I. // Mod. Phys. Lett. A. 1994. V.9. P.1735.

[10] Kosov D. S., Vdovin A. I., and Wambach J., Proc. Intern. Conf. "Nuclear Structure and Related Topics", Dubna, Sept. 9-13, 1997, eds. S. N. Ershov, R. V. Jolos, V. V. Voronov, JINR, Dubna, E4-97-327, 1997, P.254-261.

[11] Dzhioev A. A., Vdovin A. I. // Int. J. Mod. Phys. E. 2009. V.18. P.1535.

[12] Civitarese O., DePaoli A. L. // Z. Phys. A. 1993. V.344. P.243.

[13] Kosov D. S., Vdovin A. I. // Izv. RAN, ser. fiz. 1994. V.58. P.41.

[14] Goodman A. L. // Nucl. Phys. A. 1981. V.352. P.30. 